\title{
Minimally invasive thyroid anaplastic carcinoma with long survival
}

\author{
Mustafa Ümit Uğurlu' (D), İpek Erbarut Seven², Funda Eren², Cumhur Yeğen', Bahadır Mahmut Güllüoğlu
}

\begin{tabular}{|c|c|}
\hline ABSTRACT & $\begin{array}{l}\text { Anaplastic thyroid carcinoma is a highly lethal malignancy. Previously, only five cases of a noninvasive form of } \\
\text { anaplastic thyroid carcinoma with excellent prognosis were reported. We report a case of a } 68 \text {-year-old man who } \\
\text { presented with a } 3.8 \mathrm{~cm} \text { thyroid nodule diagnosed by fine needle aspiration biopsy as poorly differentiated spindled } \\
\text { and epithelial malignant cells. Lobectomy and isthmusectomy were performed, and the final diagnosis was micro- } \\
\text { invasive anaplastic thyroid carcinoma arising in a poorly differentiated carcinoma. The patient remains disease-free } \\
\text { after six years, even after hemithyroidectomy and radiation treatment. Noninvasive/microinvasive anaplastic thyro- } \\
\text { id carcinoma appears to be a different disease entity from classical anaplastic thyroid carcinoma, with favorable } \\
\text { prognosis and long disease-free survival. }\end{array}$ \\
\hline & Keywords: Thyroid, anaplastic thyroid carcinoma, microinvasion \\
\hline
\end{tabular}

ORCID ID of the author: M.Ü.U. 0000-0002-4757-5560.

Cite this paper as: Uğurlu MÜ, Erbarut Seven İ, Eren F, Yeğen C, Güllüoğlu BM. Minimally invasive thyroid anaplastic carcinoma with long survival. Turk J Surg 2018; 34(4): 319-322.

'Department of General Surgery, Marmara University School of Medicine, İstanbul, Turkey ${ }^{2}$ Department of Pathology, Marmara University School of Medicine, İstanbul, Turkey

Corresponding Author Mustafa Ümit Uğurlu e-mail: umitugurlu@gmail.com

Received: 16.09.2015 Accepted: 25.12.2015 (C) Copyright 2018 by Turkish Surgical Association Available online at turkjsurg.com

\section{INTRODUCTION}

Anaplastic (undifferentiated) thyroid carcinoma (ATC) comprises $1 \%$ to $3 \%$ of all thyroid carcinomas. It is one of the most aggressive malignancies and is characterized by extensive local invasion and rapid progression with a median survival of five months (1). According to the American Joint Committee on Cancer, all ATC patients are classified as stage IV: the primary lesion is restricted to the thyroid gland in stage IVA; involvement of the locoregional lymph nodes is observed in stage IVA/IVB; and stage IVC disease is defined by distant metastases (2). The usual course of ATC is a rapidly growing fixed neck mass that is often seen with metastatic local lymph nodes on physical examination, and $40 \%$ of patients develop multiple organ failure as cause of death (3).

A few fortunate patients with encapsulated ATC with no invasion or minimal invasion have been described in the literature (4-7). These patients have favorable outcomes and long-term survival rates compared to classical ATC patients. As noninvasive/minimal invasive anaplastic thyroid carcinoma (ATC) is a rare disease entity, we wish to share our case, who is disease-free after six years even after hemithyroidectomy and radiation treatment.

\section{CASE PRESENTATION}

A 68-year-old male was admitted for foreign body sensation in the neck. Patient had a remote history of smoking for 35 years; however, the patient had quit 15 years previously. Medical histoy of the patient consisted of coronary artery disease and chronic obstructive pulmonary disease. There was no history of radiation to the neck or thoracic region. Upon physical examination, patient had two bilateral palpable thyroid nodules. The patient was euthyroid at admission. Ultrasound (US) examination showed heterogeneous thyroid parenchyma with a $3.8 \mathrm{~cm}$ hypoechoic solid nodule on the left lobe which had fine calcification. A $2-\mathrm{cm}$ isoechoic solid nodule was observed on the right lobe with coarse calcifications. There were no cervical or thoracic radiologically significant lymph nodes. US-guided fine needle aspiration (US-FNAB) biopsy of the left thyroid nodule revealed "poorly differentiated spindled and epithelial malignant cells," and US-FNAB of the right nodule revealed a "benign colloidal nodule." No further radiological evaluation was performed. Fiber-optic laryngoscopy prior to the operation showed normal bilateral vocal cords. Total thyroidectomy was planned.

The procedure started with exploration of the left thyroid lobe, which was reported as malignant. The left thyroid lobe was mobilized with difficulty due to adhesions to the adjacent structures. A single solid nodule was palpated in that lobe. The operation was terminated with left hemithyroidectomy and isthmusectomy. The reason for terminating the operation was operative difficulties, such as adhesion to surrounding structures and concomitant bleeding. We postponed the right hemithyroidectomy and decided to wait for definitive pathological analysis of the left lobe. Postoperatively, the patient recovered without incident. 
On gross pathology, the specimen weighed 41 grams and the left lobe was $6.5 \mathrm{~cm} \times 5.5 \mathrm{~cm} \times 2.5 \mathrm{~cm}$ in diameter. On the left lobe, there was a $4 \mathrm{~cm} \times 2.2 \mathrm{~cm} \times 2 \mathrm{~cm}$ solid nodule with indistinct borders; a yellow to tan color protruded from the cut surface upon sectioning. At low magnification, the neoplasm was an encapsulated tumor showing focal follicular and insular patterns (Figure 1a, b) along with solid and trabecular patterns (Figure 1c). Focal comedo-type necrosis and mitosis were found in this poorly differentiated component. In addition, there were anaplastic areas (Figure 1a,d) composed of plump spindle cells and pleomorphic cells with vesicular chromatin and prominent nucleoli, constituting approximately $50 \%$ of the lesion. The entire tumor was submitted for histological evaluation; capsular microinvasion and vascular invasive foci (Figure 1e) were associated with this anaplastic component, although no evidence of extrathyroidal extension was obtained. The adjacent parenchyma was seen as a rim with features of mass effects of the tumor. The poorly differentiated/insular component showed positivity for cytokeratin, thyroglobulin (Figure 1f), thyroid transcription factor-1 (TTF-1) (Figure 1g), and B-cell lymphoma-2 (BCL-2) stains (Figure 1h); however, it was negative for anti-mesothelioma antibody (HBME1) stain. The anaplastic component was negative for cytokeratin, thyroglobulin (Figure 1f), TTF-1 (Figure 1g), and HBME-1. Increased staining with p53 (Figure 11) and decreased staining with BCL-2 (Figure 1j) were noted. Also, the Ki-67 (MIB-1) proliferation index was low (Figure 1k). Based on the light microscopy and immunohistochemical findings, a diagnosis of undifferentiated (anaplastic) thyroid carcinoma arising in a poorly differentiated carcinoma was reported.
During a multidisciplinary meeting, a team composed of physicians from surgery, medical oncology, radiation oncology, nuclear medicine, radiology, and pathology decided to complete the surgery as a total or near-total thyroidectomy and to administer further systemic chemotherapy and radiation therapy. The patient refused to undergo re-surgery and also refused chemotherapy; he only agreed to undergo radiation treatment. Before radiation therapy, whole body computed tomography was performed, and no evidence was found of any distant metastatic foci. At postoperative two weeks, a radiation dose of 60 Gray on a once-daily schedule was administered. The follow-up involved physical examination every three months for the first postoperative year and biannually for the following years. US examination of the neck was performed biannually. Distant metastatic screening was performed yearly with computed tomography (brain, chest, neck, and pelvis). We observed no local or distant metastasis during follow-up. The patient is disease-free 72 months after diagnosis. Recently, a positron emission tomography/computed tomography (PET/CT) scan was performed. No significant hypermetabolic lesion was observed by PET/CT imaging; only the right thyroid lobe was visible (Figure 2). The ultrasound examination of the neck confirmed the presence of a $2 \mathrm{~cm}$ isoechoic single nodule in the right lobe without changes in size or structure.

Written consent was taken from the patient to present the case report.

\section{DISCUSSION}

Anaplastic thyroid carcinoma is an extremely aggressive tumor with a disease-specific high mortality approaching 100\%. Due to its poor prognosis, ATC is classified as TNM stage IV regard-

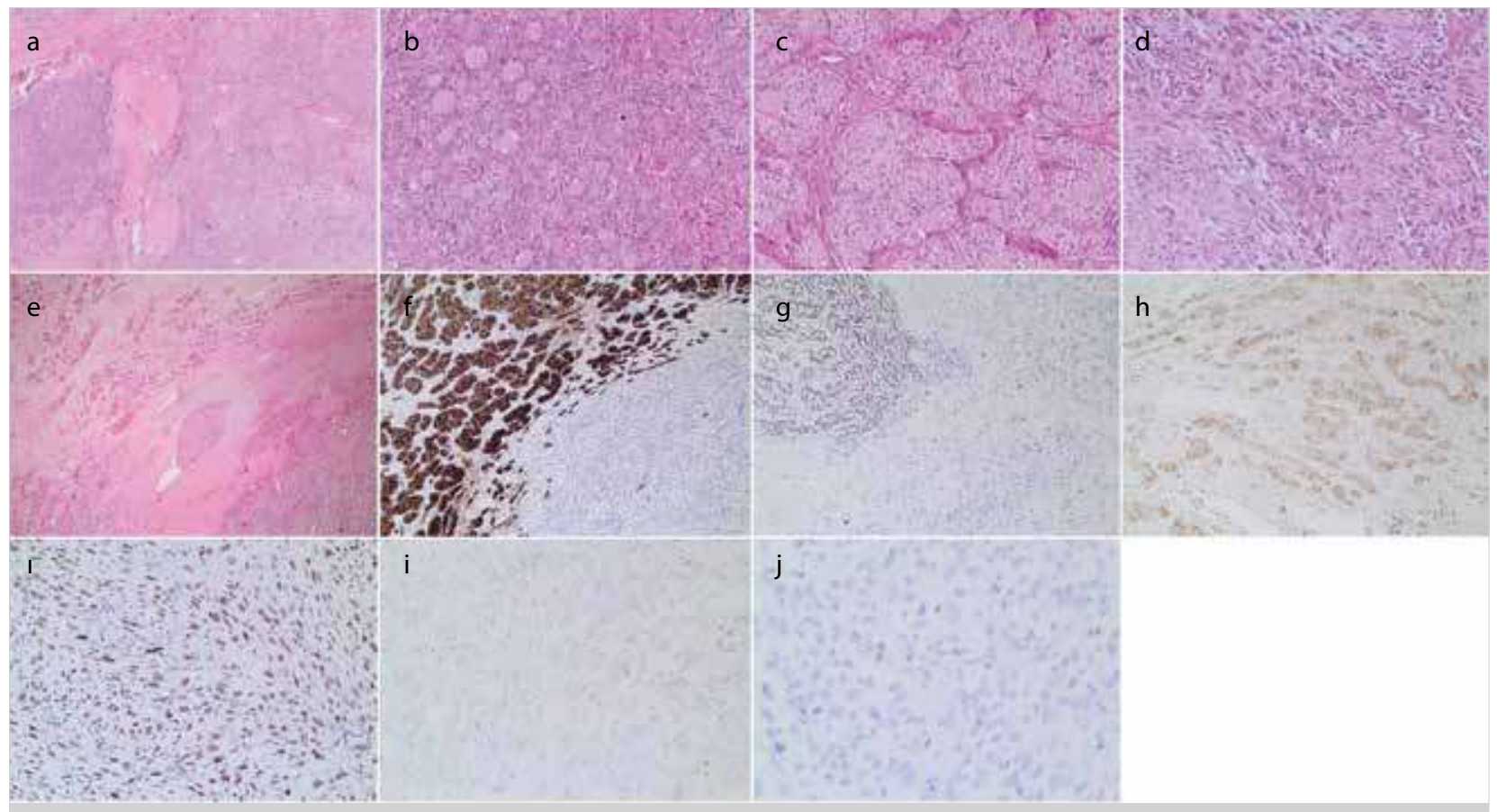

Figure 1. a-k. (a) Poorly differentiated carcinoma component (left side) and anaplastic carcinoma component (right side) (H\&E, 100x). (b) Focal follicular pattern (H\&E, 400x). (c) Trabecular pattern (H\&E, 100x). (d) Anaplastic area (H\&E, 400x). (e) Capsular and vascular invasion with anaplastic component (H\&E, 100x). (f) The follicular and trabecular component showed positivity for cytokeratins and thyroglobulin. $(\mathrm{g})$ The follicular and trabecular components showed positivity for thyroid transcription factor-1 (TTF-1). (h) The follicular and trabecular components showed positivity for BCL-2. (I) Increased staining for p53 antibody. (j) Decreased staining of the anaplastic area for BCL-2. (k) Low Ki-67 (MIB-1) proliferation index 
less of tumor burden (2). Prompt initiation of treatment is essential, and management plans, mostly for comfort care measures, should be undertaken early in the course of the disease (8).

Our case had a tumor with an ATC component; however, surprisingly, he showed an exceptional long-term disease-free survival of six years. The patient had a microinvasive anaplastic carcinoma as confirmed by conventional histology and immunohistochemistry. According to our pathologic findings, the microinvasive ATC component of the tumor demonstrated negative staining for cytokeratin, thyroglobulin TTF-1 and HBME-1, and BCL-2 and increased staining with p53; it also showed typical ATC characteristics, with high cellproliferating activity and a disrupted mechanism of apoptosis. The improved prognosis in our patient can be explained by encapsulation of the tumor. Encapsulation in ATC was previously described in a few case reports. Voutilainen et al. (5) reported a retrospective case series of ATC patients; 1 of their 33 patients had microscopic focus of ATC within follicular carcinoma and survived for 156 months. Guimaraes et al. (4) reported a 74-year-old patient with ATC who had two foci $(0.8$ $\mathrm{mm}$ and $2.5 \mathrm{~mm}$ ) of capsular invasion. Their patient remained disease-free for 35 months after total thyroidectomy. Another case from Japan, reported by Ito et al. (6) involved a 77-year old patient with a $7.5 \mathrm{~cm}$ encapsulated ATC with excellent survival. Recently, Dibelius et al. (7) reported a case of an 81-yearold man with a $3.5 \mathrm{~cm}$ encapsulated ATC. He was treated with total thyroidectomy. Even though no further adjuvant therapy was administered, the patient was disease-free 14 months after diagnosis.

Multimodal interventions involving surgery, chemotherapy, and radiation treatment are supported in current recommendations to improve both locoregional and metastatic disease outcomes (9). The decision to use these options in order or in combination must be made separately for each patient, primarily to increase the quality of life and to reduce diseasespecific mortality.

Surgery remains the cornerstone for prolonged survival in ATC when feasible. The surgical approach to ATC depends on the resectability ( $\mathrm{R} 0$ or $\mathrm{R} 1$ ) of the tumor; hence, surgical resection is recommended if it is possible and if excessive morbidity can be avoided. According to the American Thyroid Association (ATA) ATC treatment guidelines, in patients without extrathyroidal extension, total lobectomy or total/near-total thyroidectomy with therapeutic central and lateral lymph node dissection is recommended. In contrast, in patients with extrathyroidal extension, if $\mathrm{R} 1$ resection is possible, en bloc resection is recommended (10). Sugitani et al. (11) reported the data of an ATC Research Consortium of Japan (ATCCJ) cohort study of 677 patients. According to the ATCCJ, four clinical types of ATC were defined: common ATC, anaplastic change at the neck, incidental ATC, and anaplastic change at the distant site. Incidental ATC was found to have more favorable outcomes, and surgery was considered to be the main factor improving the survival. However, controversy remains regarding the effects of the extent of the surgical procedure. Venkatesh et al. (12) found that total thyroidectomy does not have any survival advantage over ipsilateral lobectomy for anaplastic tumors confined entirely to the thyroid; they also found that total thyroidectomy is associated with a higher complication rate. In a study of 134 ATC cases, neither the extent of operation nor the completeness of resection affected survival (13).

In our case, total thyroidectomy could not be technically achieved, and a probably benign thyroid lobe was left in situ. After the multidisciplinary meeting, a candid meeting was scheduled with the patient to disclose the risks and benefits of the treatment options. The options were combination treatment with completion of total/near-total thyroidectomy, adjuvant chemotherapy, and adjuvant radiation therapy. The patient refused to have completion surgery or undergo chemotherapy; his treatment was finalized with external beam radiation therapy (EBRT).

External beam radiation therapy has been suggested to reduce morbidity and mortality in adjuvant settings. After complete resection, prolonged survival ( $>2$ years) was reported in conjunction with postoperative adjuvant chemotherapy (14). High dose EBRT of $>45-50$ Gy was found to be associated with improved one-year survival; however, its toxic effects are of great concern (15). The optimal timing and the selection of the chemotherapy regimen are uncertain for classictype ATC. Non-randomized studies have evaluated different regimens concurrently with EBRT. Mostly doxorubicin-based regimens were used, and the median survival ranged from 6 months to 1 year $(16,17)$. Also, chemotherapy with a combination of taxane, anthracyclines, and cysplatin was proposed to have considerable palliative effects (18). Although combination chemotherapy was shown to improve the effects of radiation treatment in locoregional and distant disease control
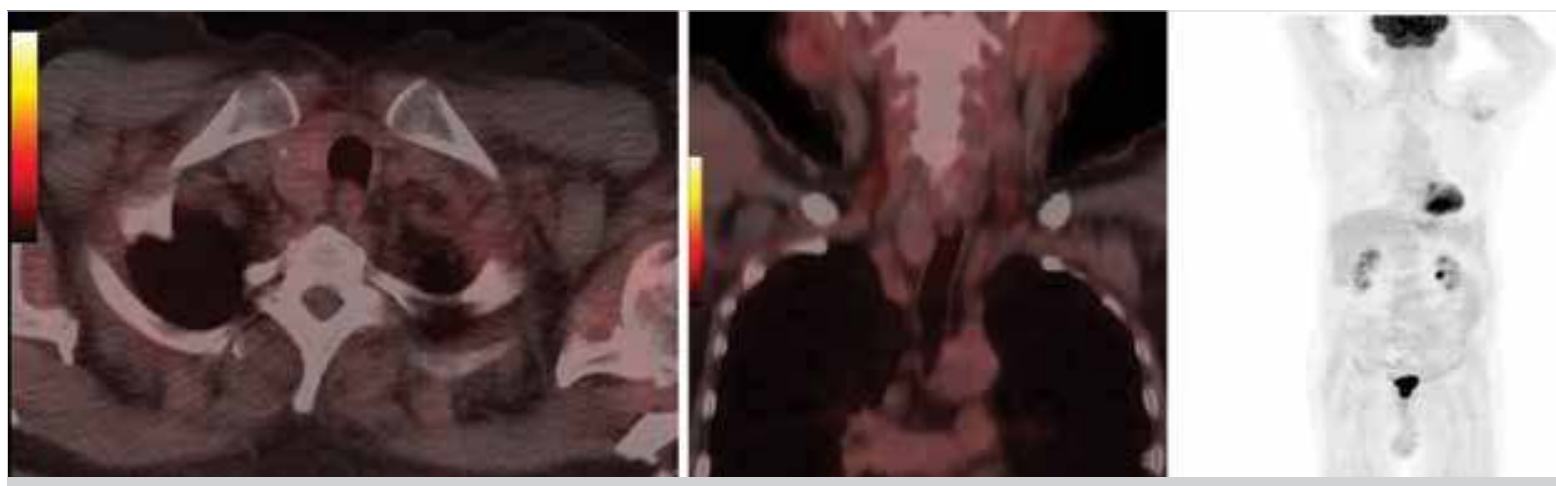

Figure 2. a-c. Positron emission tomography/computed tomography (PET/CT) imaging showing the remnant right lobe (a, b) without any area of local or distant hypermetabolic activity (c) 
in a retrospective series (19), randomized controlled trials are not available to demonstrate the benefits of combined chemotherapy. Thus, there is no standard regimen for classic-type ATC in an adjuvant setting.

\section{CONCLUSION}

Despite the overall poor outcome of classical ATC, microinvasive/noninvasive ATC may be considered to be curable. The previous five reports on this entity showed improved survival in these patients. In our case, although the histopathology revealed an encapsulated tumor with microscopic capsular and vascular invasion of anaplastic cells, the patient is a long-term survivor. There is still insufficient data regarding optimal treatment of minimally invasive or noninvasive ATC. We suggest considering reclassification of noninvasive/microinvasive ATC as a separate disease entity.

Informed Consent: Written informed consent was obtained from patient who participated in this study.

Peer-review: Externally peer-reviewed.

Author Contributions: Concept - M.U.U.; Design - M.U.U.; Supervision - F.E., C.Y., B.M.G.; Analysis and/or Interpretation - M.Ü.U., I.E.S.; Literature Search - M.Ü.U., İ.E.S.; Writing Manuscript - M.Ü.U., İ.E.S.; Critical Reviews - F.E., C.Y., B.M.G.

Conflict of Interest: The authors have no conflicts of interest to declare.

Financial Disclosure: The authors declared that this study has received no financial support.

\section{REFERENCES}

1. Brignardello E, Palestini N, Felicetti F, Castiglione A, Piovesan A, Gallo $M$, et al. Early surgery and survival of patients with anaplastic thyroid carcinoma: analysis of a case series referred to a single institution between 1999 and 2012. Thyroid 2014; 24: 1600-1606. [CrossRef]

2. Edge SB, American Joint Committee on C, American Cancer S. AJCC cancer staging handbook : from the AJCC cancer staging manual. New York: Springer; 2010.

3. Kitamura Y, Shimizu K, Nagahama M, Sugino K, Ozaki O, Mimura $\mathrm{T}$, et al. Immediate causes of death in thyroid carcinoma: clinicopathological analysis of 161 fatal cases. J Clin Endocrinol Metab 1999; 84: 4043-4049. [CrossRef]

4. Guimaraes L, Meneses A, Carrara W, Kefalas A. Encapsulated anaplastic thyroid carcinoma with three-year disease-free survival. Pathol Res Pract 2000; 196 : 867-870. [CrossRef]
5. Voutilainen PE, Multanen M, Haapiainen RK, Leppaniemi AK, Sivula AH. Anaplastic thyroid carcinoma survival. World J Surg 1999; 23: 975-998. [CrossRef]

6. Ito Y, Matsuzuka F, Yoshida H, Morita S, Nakano K, Kobayashi K, et al. Encapsulated anaplastic thyroid carcinoma without invasive phenotype with favorable prognosis: report of a case. Surg Today 2003; 33: 277-281. [CrossRef]

7. Dibelius G, Mehra S, Clain JB, Urken ML, Wenig BM. Noninvasive anaplastic thyroid carcinoma: report of a case and literature review. Thyroid 2014; 24: 1319-1324. [CrossRef]

8. Neff RL, Farrar WB, Kloos RT, Burman KD. Anaplastic thyroid cancer. Endocrinol Metab Clin North Am 2008; 37: 525-538. [CrossRef]

9. Haddad RI, Lydiatt WM, Ball DW, Busaidy NL, Byrd D, Callender G, et al. Anaplastic Thyroid Carcinoma, Version 2.2015. J Natl Compr Canc Netw 2015; 13: 1140-1150. [CrossRef]

10. Smallridge RC, Ain KB, Asa SL, Bible KC, Brierley JD, Burman KD, et al. American Thyroid Association guidelines for management of patients with anaplastic thyroid cancer. Thyroid 2012; 22: 11041139. [CrossRef]

11. Sugitani I, Miyauchi A, Sugino K, Okamoto T, Yoshida A, Suzuki S. Prognostic factors and treatment outcomes for anaplastic thyroid carcinoma: ATC Research Consortium of Japan cohort study of 677 patients. World J Surg 2012; 36: 1247-1254. [CrossRef]

12. Venkatesh YS, Ordonez NG, Schultz PN, Hickey RC, Goepfert H, Samaan NA. Anaplastic carcinoma of the thyroid. A clinicopathologic study of 121 cases. Cancer 1990; $66: 321-330$. [CrossRef]

13. Mclver B, Hay ID, Giuffrida DF, Dvorak CE, Grant CS, Thompson GB, et al. Anaplastic thyroid carcinoma: a 50-year experience at a single institution. Surgery 2001; 130: 1028-1034. [CrossRef]

14. Haigh PI, Ituarte PH, Wu HS, Treseler PA, Posner MD, Quivey JM, et al. Completely resected anaplastic thyroid carcinoma combined with adjuvant chemotherapy and irradiation is associated with prolonged survival. Cancer 2001; 91: 2335-2342. [CrossRef]

15. Bhatia A, Rao A, Ang KK, Garden AS, Morrison WH, Rosenthal DI, et al. Anaplastic thyroid cancer: Clinical outcomes with conformal radiotherapy. Head Neck. 2010;32:829-836.

16. De Crevoisier R, Baudin E, Bachelot A, Leboulleux S, Travagli JP, Caillou B, et al. Combined treatment of anaplastic thyroid carcinoma with surgery, chemotherapy, and hyperfractionated accelerated external radiotherapy. Int J Radiat Oncol Biol Phys 2004; 60: 1137-1143. [CrossRef]

17. Sherman EJ, Lim SH, Ho AL, Ghossein RA, Fury MG, Shaha AR, et al. Concurrent doxorubicin and radiotherapy for anaplastic thyroid cancer: a critical re-evaluation including uniform pathologic review. Radiother Oncol 2011; 101: 425-430. [CrossRef]

18. Foote RL, Molina JR, Kasperbauer JL, Lloyd RV, Mclver B, Morris $J C$, et al. Enhanced survival in locoregionally confined anaplastic thyroid carcinoma: a single-institution experience using aggressive multimodal therapy. Thyroid 2011;21:25-30. [CrossRef]

19. Brignardello E, Gallo M, Baldi I, Palestini N, Piovesan A, Grossi E, et al. Anaplastic thyroid carcinoma: clinical outcome of 30 consecutive patients referred to a single institution in the past 5 years. Eur J Endocrinol 2007; 156: 425-430. [CrossRef] 